\title{
Atomic-resolution Probing of Anion Migration in Perovskites with In-situ (S)TEM
}

Yu Deng ${ }^{1}$, Ruopeng Zhang ${ }^{2}$, Jim Ciston $^{3}$, Karen C Bustillo ${ }^{4}$, Colin Ophus ${ }^{5}$ and Andrew Minor ${ }^{6}$

${ }^{1}$ Nanjing University, United States, ${ }^{2}$ National Center for Electron Microscopy, Molecular Foundry, Lawrence Berkeley National Laboratory, Berkeley 94720, USA, United States, ${ }^{3}$ UC Berkeley, California, United States, ${ }^{4}$ Lawrence Berkeley National Laboratory, United States, ${ }^{5}$ Lawrence Berkeley National Laboratory, California, United States, ${ }^{6}$ UC Berkeley, Berkeley, California, United States

\section{ABSTRACT}

Perovskites are promising functional materials for their optoelectronic properties and anion migration plays a key role in their functional performance [1-3]. By using in-situ (S)TEM mechanical and electrical testing in conjunction with 4D-STEM [4,5], we directly observed/probed anion migration in perovskites at atomic resolution (see Figure 1). Here, we studied the mechanism for the anion migration in perovskites such as $(\mathrm{PbZr}) \mathrm{TiO}_{3}$ and $\mathrm{BaTiO}_{3}$, which is induced under the mechnaicl/electrical loading. To avoid the influence of the electron beam, we carried out the in-situ (S)TEM study at 60kv with low dose. And to avoid the possible strong size effect and the substrate (interface) influence, we prepared free-standing submicrometer single-crystalline structures to perform the experiments. Corresponding EDS and EELS examinations were performed to measure the local chemical change with applied stress and electrical currents. Our observations revealed the coexistence of multiple phase structures and hierarchical domain structures, as well as the greatly enhanced anion drifting and diffusion at the charged domain walls (Figure 2) and phase boundaries. The complex interaction between the local domain evolution and phase transition has been discussed. Based on above investigations, a model for anion migration in perovskire under mechanical/electrical loading has been presented.

\section{ACKNOWLEDGEMENTS}

The authors acknowledge support by the National Natural Science Foundation of China (Grants Nos. 50802039) and Natural Science Foundation of Jiangsu Province, China (Grant Nos. BK20151382), and the National Science Foundation through the STROBE Science and Technology Center. RZ acknowledges funding from the US Office of Naval Research under Grant No. N00014-12-1-0413 and N00014-17-12283. The electron microscopy work was performed at the Molecular Foundry, Lawrence Berkeley National Laboratory, which is supported by the U.S. Dept. of Energy under Contract \# DE-AC0205CH11231. J.C. Acknowledges additional support from the Department of Energy Early Career Research Program.

References

1. G. Catalan, J. Seidel, R. Ramesh, J. F. Scott, Rev. Modern Phys. 84, 119 (2012).

2. Y. Deng, C. Gammer, J. Ciston, P. Ercius, C. Ophus, K. Bustillo , C. Song, R. Zhang, D. Wu, Y. Du, Z. Chen, H. Dong, A. G. Khachaturyan, A. M. Minor, Acta Materialia 181, 501 (2019).

3. J. Burschka, N. Pellet, S. J. Moon, R. Humphry-Baker, P. Gao, M. K. Nazeeruddin, M. Gratzel, Nature 499, $316(2013$.

4. C. Gammer, V. B. Ozdol, C.H. Liebscher, and A.M. Minor, Ultramicroscopy, 155, 1 (2015).

5. V.B. Ozdol, C. Gammer, X.G. Jin, P. Ercius, C. Ophus, J. Ciston, A.M. Minor, Applied Physics Letters, 106, 253107 (2015). 

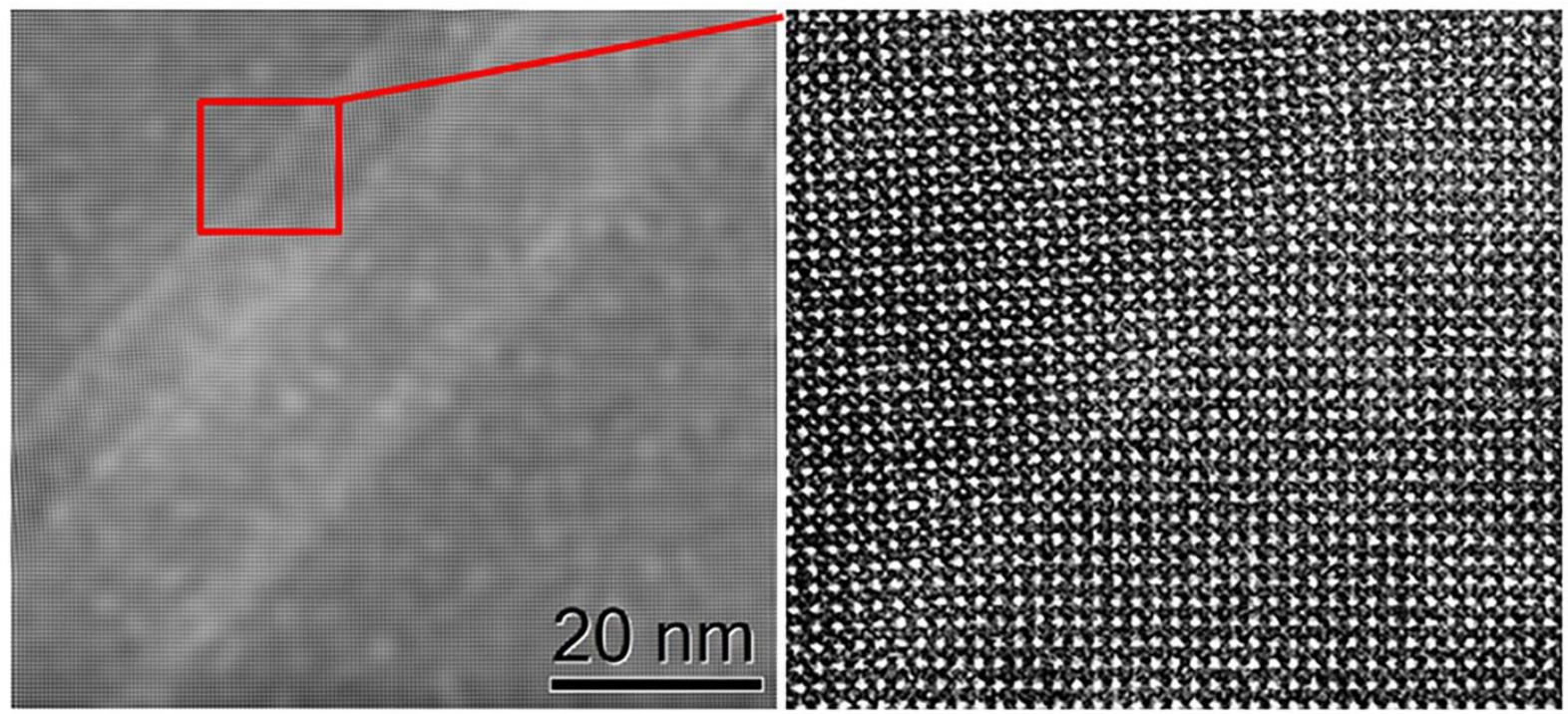

Figure 1. Figure 1 The in-situ atomic-resolution observation of a $(\mathrm{PbZr}) \mathrm{TiO} 3$ under compression loading, where anion migration is induced at domain wall.

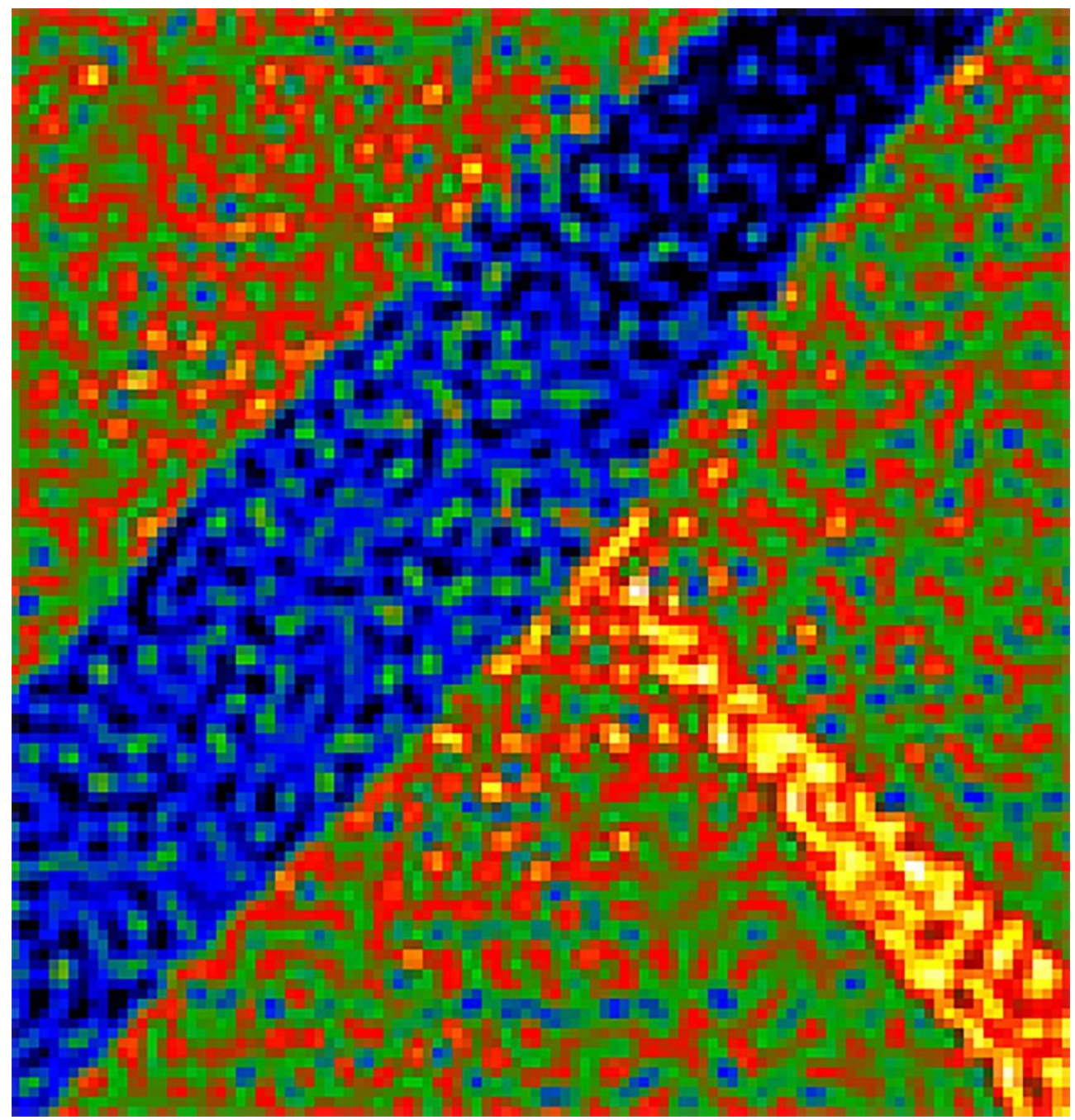

Figure 2. Figure 2 The 4D-STEM mapping on the charged domain wall in (PbZr)TiO3 